\title{
Решетки четырехзначных модальных логик
}

\author{
Карпенко Александр Степанович \\ Сектор логики, Институт философии РАН. \\ 119991, Россия, Москва, ул. Волхонка, 14, строение 5. \\ e-mail: as.karpenko@gmail.com
}

В статье рассматриваются четыре решетки четырехзначных модальных логик. В основе построения лежат различные алгебраические структуры, которые затем последовательно расширяются эндоморфизмами и константными функциями. В первом случае строится решетка расширений булевой алгебры $B^{2}$, затем строится решетка расширений алгебры Де Моргана $D M 4$. В обоих случаях возникают различные модальные логики, свойства которых описываются и сравниваются между собой. Отдельно рассматривается решетка, где появляется тетравалентная модальная логика TML. Наконец, первые две решетки «объединяются» и вычленяется класс основных

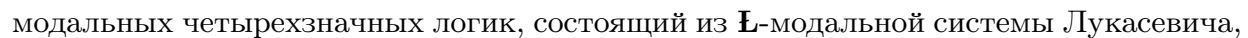
логики Собочиньского V2 и логики истины фон Вригта $\mathbf{T}^{\prime \prime}$. Особого внимания заслуживает логика $\mathbf{T r}$, которая функционально эквивалентна логике $\mathbf{V 2}$, и занимает центральное место в последней решетке. Она единственная из всех рассмотренных четырехзначных модальных логик, которая обладает интерполяционным свойством Крейга, и кроме того, является прекрасным кандидатом на роль пропозициональной логики истины. В заключение в статье представлена ее аксиоматизация.

Keywords: модальные логики, решетки логик, булева алгебра, алгебра Де Моргана, эндоморфизмы, замкнутые классы функций, булевы каскады, логика $\mathbf{T r}$

\section{1 Введение}

Я. Лукасевич в середине прошлого века сделал следующее заявление: «Я стою на той точке зрения, что в любой модальной логике должно быть сохранено классическое исчисление предложений. До сих пор это исчисление продемонстрировало свою надежность и полезность, и оно не должно быть отвергнуто без достаточно веских оснований» $[2$, c. 233]. В результате, Лукасевич отказывается от своей знаменитой трехзначной логики $\mathbf{E}_{3}$ и ее модального варианта и строит четырехзначную $\mathbf{L}$-модальную систему $[23]^{1}$, к которой мы еще вернемся.

Однако на самом деле все началось с классической книги К. Льюиса и К. Лэнгфорда [22], где определяются и исследуются модальные системы $\mathbf{S} 1-\mathbf{S} 5$, используя главным образом 4-значные матрицы. Здесь

\footnotetext{
${ }^{1} \mathrm{O}$ философских основаниях этих логик и их модальных свойствах см. [8].
} 
было выделено пять групп четырехзначных матриц, которые различались лишь определением оператора необходимости $\square$, в то время как немодальная часть представляет собой не что иное, как четырехзначную классическую логику $\mathbf{C}_{4}$.

\section{2 Четырехзначная классическая логика $\mathrm{C}_{4}$}

Пусть $\mathfrak{M}_{4}^{C}$ есть четырехзначная логическая матрица

$$
<M, \rightarrow, \vee, \wedge, \neg,\{1\}>,
$$

где $M=\{1, a, b, 0\}$. Эта матрица получена посредством прямого произведения матрицы $\mathfrak{M}_{2}^{C}$ (для классической пропозициональной логики $\mathbf{C}_{2}$ ) саму на себя, т.е. $\mathfrak{M}_{4}^{C}=\mathfrak{M}_{2}^{C} \times \mathfrak{M}_{2}^{C}$, где матричные операции $\neg, \rightarrow, \vee, \wedge$ определяются следующим образом:

\begin{tabular}{|c|c|}
\hline$x$ & $\neg x$ \\
\hline 1 & 0 \\
$a$ & $b$ \\
$b$ & $a$ \\
0 & 1 \\
\hline
\end{tabular}

\begin{tabular}{|c|cccc|}
\hline$\rightarrow$ & 1 & $a$ & $b$ & 0 \\
\hline 1 & 1 & $a$ & $b$ & 0 \\
$a$ & 1 & 1 & $b$ & $b$ \\
$b$ & 1 & $a$ & 1 & $a$ \\
0 & 1 & 1 & 1 & 1 \\
\hline
\end{tabular}

\begin{tabular}{|c|cccc|}
\hline$\vee$ & 1 & $a$ & $b$ & 0 \\
\hline 1 & 1 & 1 & 1 & 1 \\
$a$ & 1 & $a$ & 1 & $a$ \\
$b$ & 1 & 1 & $b$ & $b$ \\
0 & 1 & $a$ & $b$ & 0 \\
\hline
\end{tabular}

\begin{tabular}{|l|llll|}
\hline$\wedge$ & 1 & $a$ & $b$ & 0 \\
\hline 1 & 1 & $a$ & $b$ & 0 \\
$a$ & $a$ & $a$ & 0 & 0 \\
$b$ & $b$ & 0 & $b$ & 0 \\
0 & 0 & 0 & 0 & 0 \\
\hline
\end{tabular}

Обратим внимание, что здесь $M$ - частично упорядоченное множество: $0<b, a<1$ (то есть $b$ и $a$ несравнимы).

Хорошо известно, что матрица $\mathfrak{M}_{4}^{C}$ является характеристической для $\mathbf{C}_{2}$. Логику с соответствующими логическими связками обозначим посредством $\mathbf{C}_{4}$. Ее алгебраическим эквивалентом является четырехэлементная булева алгебра $B^{2}=B \times B(B-$ двухэлементная булева алгебра).

\section{3 Эндоморфизмы}

Заметим, что имеется всего 256 унарных четырехзначных операторов, которыми можно расширить $\mathbf{C}_{4}$. Было бы желательно иметь какойто критерий для их выбора с целью построения наиболее интересных 
модальных четырехзначных логик. В 1966 г. Э. Леммон (см. [10]) выделяет 15 унарных операторов, которые добавляются к $\mathbf{C}_{4}$. Все эти 15 групп матриц называются регулярными, поскольку они верифицируют модальную аксиому $\mathbf{K}: \square(\varphi \supset \psi) \supset(\square \varphi \supset \square \psi)^{2}$.

Однако имеется одно очень важное и весьма эффективное ограничение на выбор этих операторов, предложенное в работе Н.М. Ермолаевой и А.А. Мучника [7]. Еще в статье [6, с. 229] они обратили внимание на то, что модальные операторы, а также временные операторы в ряде модальных (временных) логик и соответствующих алгебр выражаются с помощью эндоморфизмов в дистрибутивных решетках.

Фундаментальную роль в дальнейшем будут играть одноместные функции $g, e_{1}$ и $e_{2}$, задаваемые таблично:

\begin{tabular}{|c|c|c|c|}
\hline$x$ & $g(x)$ & $e_{1}(x)$ & $e_{2}(x)$ \\
\hline 1 & 1 & 1 & 1 \\
$a$ & $b$ & 0 & 1 \\
$b$ & $a$ & 1 & 0 \\
0 & 0 & 0 & 0 \\
\hline
\end{tabular}

В булевой алгебре $B^{2}$ эти функции являются эндоморфизмами:

$$
\begin{aligned}
& f(x \vee y)=f(x) \vee f(y), f(x \wedge y)=f(x) \wedge f(y), \\
& f(\neg x)=\neg f(x), f(1)=1, f(0)=0, f\left(x^{\sigma}\right)=(f(x))^{\sigma}, 3
\end{aligned}
$$

где $f$ может быть любой из функций $g, e_{1}$ и $e_{2}$. В [7] отмечается, что вместе с $e_{0}(x)=x$ (тождественной функцией) $g, e_{1}$ и $e_{2}$ образуют моноид $Q$ всех эндоморфизмов $B^{2}$. Если наряду с эндоморфизмами взять также константные функции $a(x)=a, b(x)=b, 1(x)$ и $0(x)=0$, то вместе с $g, e_{1}$ и $e_{2}$ они образуют моноид $P$ всех эндоморфизмов $B^{2}$ как дистрибутивной решетки, которые могут не сохранять 0 и 1.

Основной результат статьи [7] состоит в описании всех функционально замкнутых классов на $M$, т.е. функций из $P_{4}\left(P_{4}\right.$ есть класс всех

\footnotetext{
${ }^{2}$ В этой работе Леммон также описывает двухэлементные модели Крипке для всех 15 четырехзначных модальных логик, являющихся расширением $\mathbf{C}_{4}$ (с. 121).

$$
x^{\sigma}=\left\{\begin{array}{l}
x, \text { при } \sigma=1 \\
\neg x, \text { при } \sigma=0 .
\end{array}\right.
$$
}


четырехзначных функций логики Поста [6]), содержащих булевы функции $\vee, \wedge$ и $\neg$. Приводится решетка этих классов и для минимальных расширений определены соответствующие четырехзначные модальные логики ${ }^{4}$.

\section{4 Решетка расширений булевой алгебры $B^{2}$}

Обозначим через $\left(f_{1}, f_{2}, \ldots, f_{k}\right)$ функционально замкнутый класс, порождаемый функциями $\left(f_{1}, f_{2}, \ldots, f_{k}\right)$ из $P_{4}$ и положим

$$
\begin{aligned}
& B^{2}=(\vee, \wedge, \neg) ; \quad P_{g}=(\vee, \wedge, \neg, g) ; \quad P_{1}=\left(\vee, \wedge, \neg, e_{1}\right) ; \\
& P_{2}=\left(\vee, \wedge, \neg, e_{2}\right) ; \quad P_{a b}=(\vee, \wedge, \neg, a)=(\vee, \wedge, \neg, b) ; \\
& P_{a b 1}=\left(\vee, \wedge, \neg, e_{1}, a, b\right) ; \quad P_{a b 2}=\left(\vee, \wedge, \neg, e_{2}, a, b\right) ; \\
& P_{12}=\left(\vee, \wedge, \neg, e_{1}, e_{2}\right)=T_{01} .
\end{aligned}
$$

В [7] доказано, что $P_{4}=\left(\vee, \wedge, \neg, e_{1}, e_{2}, a, b\right)$ и что все замкнутые (функционально) расширения $B^{2}$ выписаны выше. Решетка этих классов указана на рис. 1 (см. [7, с. 301]):

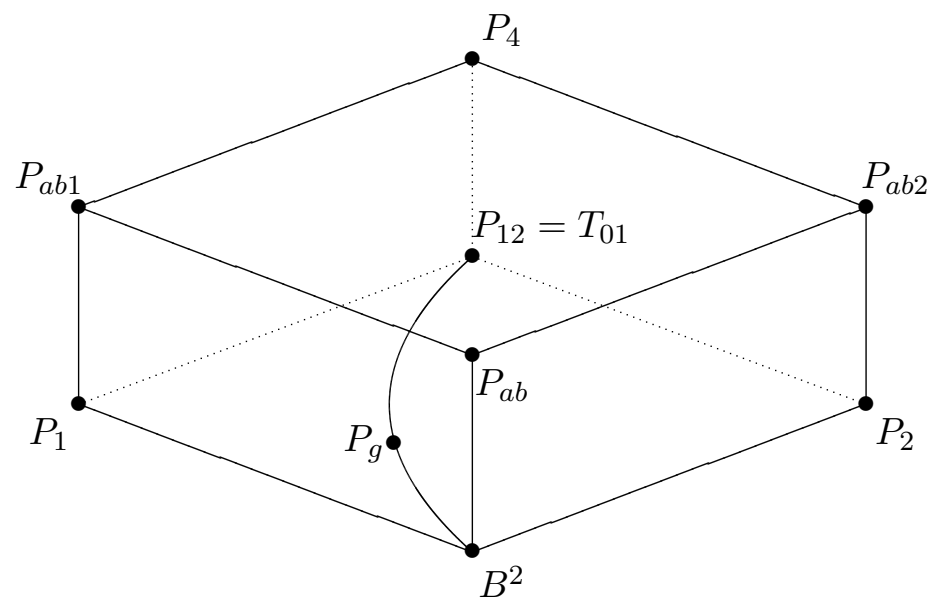

Рис. 1.

\footnotetext{
${ }^{4} \mathrm{~B}$ связи с построением решеток замкнутых (функционально) классов конечнозначных функций обратим внимание на работу Н.Е. Томовой [4], где строится изящная семиэлементная решетка расширений трехзначной слабой логики Клини «естественными» импликациями. См. также [5].
} 
Обратим внимание на то, что все минимальные расширения $B^{2}-$ $P_{g}, P_{1}, P_{2}, P_{a b}$ (логики первого уровня) функционально эквивалентны модальным логикам: $P_{g}$ - логике Собочиньского $\mathbf{V} 2, P_{1}$ и $P_{2}-$ логике Собочиньского $\mathbf{K} 4$, которую, чтобы отличить от льюисовской модальной системы $\mathrm{K} 4$, будем обозначать посредством $\mathrm{K} 4(\mathrm{~S})$, и $P_{a b}-$ $\mathbf{L}$-модальной системе Лукасевича, упомянутой нами выше. Рассмотрим эти модальные логики по отдельности, а затем определимся с системой $P_{12}$.

Модальная логика Собочиньского V2 есть расширение S5 посредством формулы:

$$
\square \varphi \vee \square(\phi \rightarrow \psi) \vee \square(\varphi \rightarrow \neg \psi)[25, \text { c. 305], }
$$

где модальный оператор $\square$ определяется следующим образом:

$$
\square 1=1 ; \square a=\square b=\square 0=0 .
$$

Модальный оператор с такими свойствами назовем «стандартным». Характеристической матрицей для $\mathbf{V} \mathbf{2}$ со связками $\rightarrow$, $\neg$ и $\square$ как раз является матрица «группы III» из [22, с. 493].

Модальная логика Собочиньского K4(S) [26] есть расширение $\mathbf{S 4 . 4}$ посредством формулы $\square(\square \diamond \varphi \rightarrow \diamond \square \psi)$, где $\mathbf{S 4 . 4}$ есть $\mathbf{S} 4+\square(\varphi \rightarrow$ $(\diamond \square \varphi \rightarrow \square \varphi)$ ), и модальный оператор $\square$ определяется следующим образом:

$$
\square 1=1 ; \square a=0 ; \square b=b ; \square 0=0 .
$$

В [27] было доказано, что матрица со связками $\rightarrow, \neg$ и $\square$ (матрица «группы II» из [22, с. 493]) является характеристической для модальной логики $\mathrm{K} 4(\mathrm{~S})$.

Общепринятая аксиоматизация $\mathbf{\mathbf { L } - м о д а л ь н о и ̆ ~ с и с т е м ы ~ Л у к а с е в и ч а ~}$ представлена в [1, с. 160]:

1. Классическая пропозициональная логика $\mathbf{C}_{2}$.

2. $\square(\varphi \rightarrow \psi) \rightarrow(\square \varphi \rightarrow \square \psi)$,

3. $\square \varphi \rightarrow \varphi$,

4. $\square \varphi \rightarrow(\psi \rightarrow \square \psi)$,

где оператор $\square$ определяется следующим образом:

$$
\square 1=\square a=a ; \square b=\square 0=0 .
$$


Во всех рассмотренных системах два правила вывода: modus ponens и правило Гёделя.

Интересна проблема аксиоматизации непосредственно систем $P_{g}=$ $(\vee, \wedge, \neg, g), P_{1}=\left(\vee, \wedge, \neg, e_{1}\right), P_{2}=\left(\vee, \wedge, \neg, e_{2}\right)$ и $P_{a b}=(\vee, \wedge, \neg, a)=$ $(\vee, \wedge, \neg, b)$. K решению это проблемы для $P_{g}$ вернемся в конце статьи.

Рассмотрим теперь логики второго уровня: $P_{a b 1}, P_{a b 2}$ и $P_{12}$. Две логики, являющиеся соответственно расширением $\mathbf{L}$-модальной системы Лукасевича эндоморфизмами $e_{1}$ и $e_{2}$, появляются впервые.

Относительно $P_{12}$ в [7] лишь указывается, что этот класс функций соответствует предполному ${ }^{5}$ в $P_{4}$ классу функций $T_{01}$, сохраняющих $\{1,0\}$ (см. [6]). Показано, что класс $T_{01}$ порождается базисом $\left\{\vee, \wedge, \neg, e_{1}, e_{2}\right\}$, а также $\left\{\vee, \wedge, \neg, g, e_{k}\right\}(k=1,2)$, то есть равен $P_{12}{ }^{6}$ Однако заметим, что из теоремы B.К. Финна [3] о функциональных свойствах многозначных логик Лукасевича $\mathbf{L}_{n}$ следует, что $\mathbf{L}_{4}$ по своим функциональным свойствам соответствует классу Яблонского $T_{01}$. Таким образом, расширение $P_{12}$ функционально эквивалентно четырехзначной логике Лукасевича $\mathbf{L}_{4}$.

Все эти рассуждения опирается на довольно-таки громоздкие результаты. Более непосредственно это выглядит следующим образом. Пусть $0<b<a<1$, то есть $M$ линейно упорядочено. Покажем что классы $\left\{\vee, \wedge, \neg, e_{1}, e_{2}\right\}$ и $\left\{\cup, \cap, \neg, J_{1}, J_{a}, J_{b}, J_{0}\right\}$ функционально эквивалентны, где $x \cup y=\max (x, y), x \cap y=\min (x, y)$ и $J_{i}$-функции выразимы в $P_{12}$ следующим образом:

$$
\begin{aligned}
& J_{1}(x)=e_{1}(x) \wedge e_{2}(x), \\
& J_{a}(x)=\neg e_{1}(x) \wedge e_{2}(x), \\
& J_{b}(x)=e_{1}(x) \wedge \neg e_{2}(x), \\
& J_{0}(x)=\neg e_{1}(x) \wedge \neg e_{2}(x) .
\end{aligned}
$$

Теперь, используя $J_{i}$-функции, можно выразить $x \cup y$ :

$x \cup y=(x \wedge y) \vee\left(J_{0}(x) \wedge y\right) \vee\left(x \wedge J_{0}(y)\right) \vee\left(J_{b}(x) \wedge y\right) \vee\left(x \wedge J_{b}(y)\right) \vee J_{1}(x) \vee J_{1}(y)$, $x \cap y=\neg(\neg x \cup \neg y)$.

\footnotetext{
${ }^{5}$ Система $F$ функций называется предполной в $P_{n}$, если $F$ представляет не полную систему, но добавление к $F$ любой функции $f$ такой, что $f \in P_{n}$ и $f \notin F$ преобразует $F$ в полную систему. Подробно о функциональных свойствах конечнозначныхлогик см. [9, гл. 7].

6 Это также означает, что «дуга» на рис. 1 от $B^{2}$ к $P_{12}$ правильно показывает, что класс $P_{12}$ содержит эндоморфизм $g$.
} 
Таким образом, классы $\left\{\vee, \wedge, \neg, e_{1}, e_{2}\right\}$ и $\left\{\cup, \cap, \neg, J_{1}, J_{a}, J_{b}, J_{0}\right\}$ функционально эквивалентны. Остается только заметить, что класс $\left\{\cup, \cap, \neg, J_{1}, J_{a}, J_{b}, J_{0}\right\}$ является базисом для четырехзначной логики Лукасевича $\mathbf{L}_{4}$ (см. [9, с. 125]).

Что касается самого класса $P_{4}$, то его функциональная полнота следует из того, что, как уже говорилось, класс $T_{01}$ предполон в $P_{4}$, и этот класс обладает свойством сохранения 0 и 1 . Поэтому добавление к этому классу функций $a$ или $b$, не обладающих этим свойством, обеспечивает функциональную полноту $P_{4}$.

\section{5 Четырехзначная логика Белнапа $\mathrm{B}_{4}$}

В [1] и [2] Н. Белнапом была предложена «полезная четырехзначная логика» (будем обозначать ее посредством $\mathbf{B}_{4}$ ), которая со временем вызвала необычайный интерес, особенно среди специалистов в области информатики и искусственного интеллекта. Логическими связками $\mathbf{B}_{4}$ являются $\vee, \wedge$ и $\sim$, где отрицание $\sim$ определяется следующим образом: $\sim 1=0 ; \sim a=a ; \sim b=b ; \sim 0=1$.

Алгебры, соответствующие логике Белнапа $\mathbf{B}_{4}$, стали называться решетками Де Моргана ${ }^{7}$ и обозначаться посредством $D M 4$. Тщательному изучению $\mathbf{B}_{4}$ посвящена статья [8], где представлено элегантное секвенциальное исчисление для $\mathbf{B}_{4}$, его взаимоотношение с подобными исчислениями для трехзначной логики Клини $\mathbf{K}_{3}$ и для классической логики $\mathbf{C}_{2}$. Главный результат заключается в следующем: класс решеток Де Моргана является алгебрачческим примером $\mathbf{B}_{4}$; точно так же, как класс булевых алгебр является алгебраическим примером $\mathbf{C}_{2} \cdot{ }^{8}$

Четырехзначная логика Белнапа $\mathbf{B}_{4}$ оказалась очень полезной в качестве базиса для других логик. Особенно интересны ее расширения стандартным модальным оператором $\square$ (см. выше логику V2) и связкой импликации ${ }^{9}$. Однако вначале опишем функционально замкнутые классы на $M$, содержащие функции $\vee, \wedge$ и $\sim$, и рассмотрим расширения решетки Де Моргана DM4.

\footnotetext{
${ }^{7}$ Решеткой Де Моргана называется дистрибутивная решетка $<A, \vee, \wedge>$ с операцией $\sim$, причем $\sim(\mathrm{x} \vee \mathrm{y})=\sim x \wedge \sim y ; \sim(x \wedge y)=\sim x \vee \sim y ; \sim \sim x=x$. Операция $\sim$ называется отрицанием Де Моргана. Решетка Де Моргана с 1 и 0 называется алгеброй Де Моргана, причем $a \vee b=1 ; a \wedge b=0$.

${ }^{8} \mathrm{Cм}$. также [24].

${ }^{9}$ См. четырехзначную логику В.М. Попова Par [3], которая представляет собой расширение $\mathbf{B}_{4}$ соответствующей импликацией. Здесь дается секвенциальная и гильбертовская аксиоматизация Par. В [24] исследуется алгебраический эквивалент логики Par, который называется«импликативной алгеброй Де Моргана».
} 


\section{6 Решетка расширений DM4}

Как и ранее, обозначим через $\left(f_{1}, f_{2}, \ldots, f_{k}\right)$ функционально замкнутый класс, порождаемый функциями $f_{1}, f_{2}, \ldots, f_{k}$ из $P_{4}$ и положим:

$$
\begin{aligned}
& D M 4=(\vee, \wedge, \sim) ; \quad P_{g}=(\vee, \wedge, \sim, g) ; \\
& P_{e_{1} e_{2}}=\left(\vee, \wedge, \sim, e_{1}\right)=\left(\vee, \wedge, \sim, e_{2}\right)=P_{\oplus} ; \\
& P_{a}=(\vee, \wedge, \sim, a) ; \quad P_{b}=(\vee, \wedge, \sim, b) ; \\
& P_{\oplus a}=\left(\vee, \wedge, \sim, e_{1}, e_{2}, a\right) ; \quad P_{\oplus b}=\left(\vee, \wedge, \sim, e_{1}, e_{2}, b\right) ; \\
& P_{a b}^{\prime}=(\vee, \wedge, \sim, a, b)^{10} .
\end{aligned}
$$

Покажем, что $\left(\vee, \wedge, \sim, e_{1}, e_{2}, a, b\right)=\left(\vee, \wedge, \neg, e_{1}, e_{2}, a, b\right)=P_{4}$ :

$$
\begin{aligned}
& g(x)=\left(b \wedge e_{1}(x)\right) \vee\left(a \wedge e_{2}(x)\right)(\text { см. [7, с. 302]), } \\
& \neg(x)=g \sim(x) .
\end{aligned}
$$

Решетка выписанных выше классов указана на рис. 2:

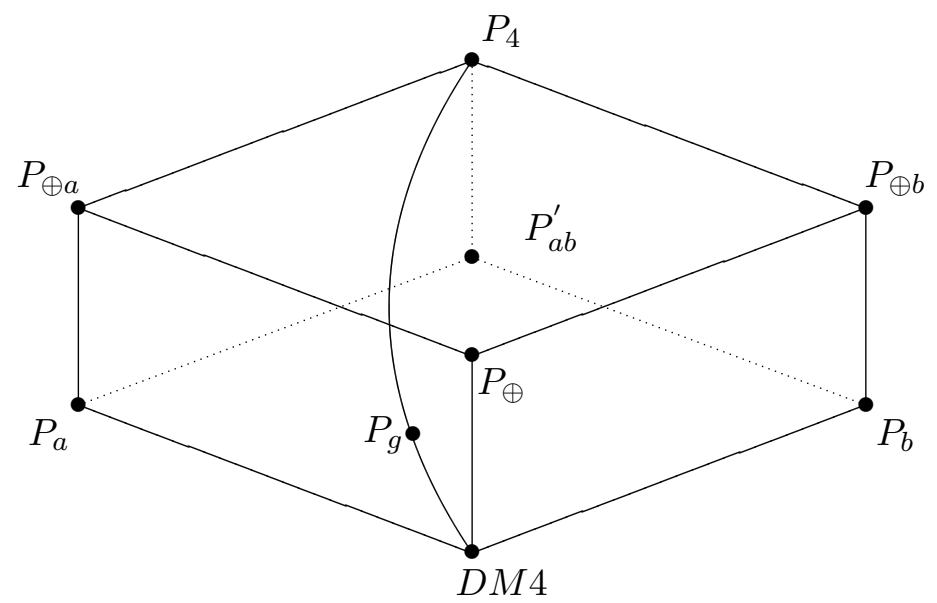

Рис. 2.

Вначале покажем, что логики со связками $(\vee, \wedge, \neg, g)$ и $(\vee, \wedge, \sim, g)$ функционально эквивалентны:

$$
\neg(x)=g \sim(x) \text { и } \sim(x)=g \neg(x) .
$$

\footnotetext{
${ }^{10}$ Здесь $P_{a b}^{\prime}$, чтобы отличить ее от $P_{a b}$ на рис. 1 .
} 
Поэтому имеем $P_{g}$ как в решетке на рис. 1 , так и в решетке на рис. 2 . В дальнейшем логику со связками $(\vee, \wedge, \neg, g)$ будем обозначать посредством Tr.

В этой решетке особый интерес представляет логика со связками $\left(\vee, \wedge, \sim, e_{1}, e_{2}\right)$, которая есть не что иное как логика истины $\Gamma$. фон Вригта [4], обозначаемая посредством $\mathbf{T}^{\prime \prime} \mathbf{L M}$ (в дальнейшем $\mathbf{T}^{\prime \prime}$ ). Подробно об ее свойствах и аксиоматизации см. в [21]. Заметим только, что в ней эндоморфизмы $e_{1}$ и $e_{2}$ взаимовыразимы:

$$
e_{1}(x)=: \sim\left(e_{2}(\sim x)\right) \text { и } e_{2}(x)=: \sim\left(e_{1}(\sim x)\right) .
$$

\section{7 Тетравалентная модальная логика TML}

В приведенной выше решетке не нашлось места для модальной логики TML, которая привлекла к себе большое внимание, особенно со стороны алгебраистов. Строится она аналогично тому, как была получена модальная логика V2. В первом случае, булева алгебра расширяется стандартным модальным оператором $\square$, а во втором - этим же оператором расширяется алгебра Де Моргана.

Впервые логика $\mathbf{B}_{4}$, расширенная этим модальным оператором, изучалась в [5], где был также представлен ее алгебраический эквивалент с теоремой представления. Здесь отмечается, что алгебра подобной логики возникла еще при первоначальной попытке $Г$. Мойсила найти короткую систему аксиом для трехэлементных алгебр Лукасевича.

Специально изучению такой логики под названием TML (tetravalent modal logic) и их алгебрам (TMA) посвящена обстоятельная статья [9] (см. также [7]). Здесь отмечается, что $T M A$ первоначально изучались А. Монтейро при исследовании трехэлементных алгебр Лукасевича.

В [9] приводится изящная аксиоматизация $T M A$, к аксиомам алгебры Де Моргана добавляются следующие две аксиомы:

$$
\begin{aligned}
& \text { (TM1) } \square x \wedge \sim x=0, \\
& (\mathrm{TM} 2) \sim \square x \wedge x=\sim x \wedge x .
\end{aligned}
$$

Здесь же представлено секвенциальное исчисление логики TML (c. 496).

Заметим, что логики $\operatorname{Tr}$ и $\mathbf{T}^{\prime \prime}$ являются расширением TML, т.е. через операции $\operatorname{Tr}$ (через операции $\mathbf{T}^{\prime \prime}$ ) выразим модальный оператор $\square$. Покажем это:

1) $\square x=x \wedge g(x)$,

2) $\square x=e_{1}(x) \wedge e_{2}(x)$. 
Теперь мы можем представить еще одну решетку расширений $D M 4$, используя на этот раз только эндоморфизмы и модальный оператор $\square x:$

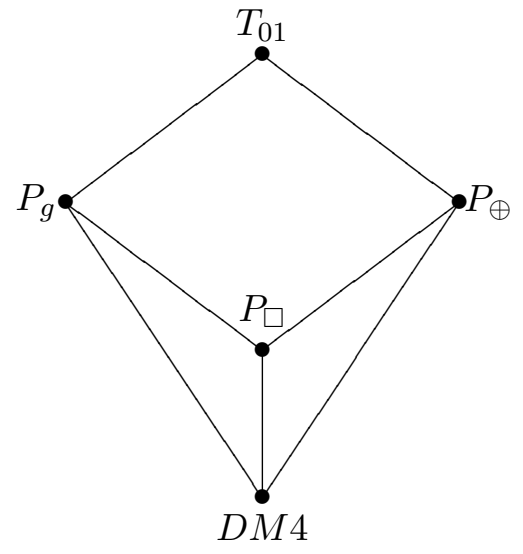

Рис. 3.

Здесь класс $P_{\square}$ есть $(\vee, \wedge, \sim, \square)$, который в точности соответствует логическим операциям логики TML.

\section{8 Основная решетка четырехзначных модальных логик}

Наша задача - по возможности объединить в единую решетку логики, представленные классами функций на рис. 1 и на рис. 2. Пусть $D$ есть дистрибутивная решетка $D=(\vee, \wedge)$, которая на рис. 4 расширяется слева операциями $\neg$ и $a, b$, а справа операциями $\sim$ и $e_{1}, e_{2}$ :

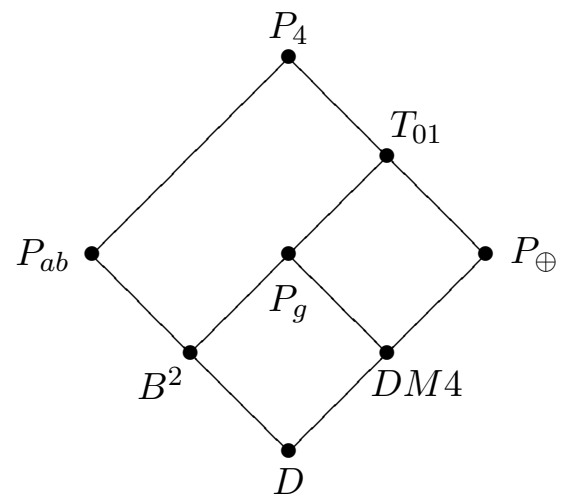

Рис. 4. 
Обратим внимание на центральный ряд - это расширения $P_{a b}, P_{g}$ и $P_{\oplus}$, которые функционально эквивалентны следующим модальным логикам: $\mathbf{L}$-модальной системе Лукасевича, логике $\operatorname{Tr}$ и логике фон Вригта $\mathbf{T}^{\prime \prime}$. Эти модальные логики назовем фундаментальными. Особое место здесь по праву занимает логика $\mathrm{Tr}$.

\section{9 Булева склейка}

Однако имеется более естественный путь объединения этих двух решеток в одну. Обратим внимание на то, что при пересечении классов функций обеих решеток совпадают только два класса $-P_{4}$ и $P_{g}$. Таким образом, объединение двух решеток происходит следующим образом: вершина решетки на рис. 2 «склеивается» с вершиной решетки на рис. 1 , то есть «склеиваются» два класса $P_{4}$, а класс $P_{g}$ является тем «стержнем», который связывает обе решетки. В итоге имеем 16-ти элементную решетку, которая является результатом «склейки» двух 8-ми элементных булевых решеток. Полученная решетка заслуживает специального исследования, что может послужить основанием для введения нового класса алгебраических структур.

В связи с этим обратим внимание на алгебраические структуры под названием «булевы каскады», введенные Л. Эсакиа в [16]. Булев каскад - это алгебра Гейтинга, изоморфная каскадному соединению булевых решеток. В этом соединении наибольший элемент предшествующей булевой решетки $B_{i}$ является наименьшим элементом последующей булевой решетки $B_{i+1}$. Булевы каскады являются алгебраическими моделями интуиционистской логики $\mathbf{H}$, в которой верен слабый закон Пирса $\mathbf{w P}$, то есть формула

$$
(q \Rightarrow p) \vee[((p \Rightarrow q) \Rightarrow p) \Rightarrow p] .
$$

В [8] представлено два примера булевых каскадов (соединение двух 8-ми элементных булевых решеток), элементами каждого из которых являются импликативные логики.

В связи с вышесказанным возникает вопрос об алгебраической структуре нашей булевой «склейки», и алгебраическим примером какой логики эта «склейка» является?

\section{0 Модальная логика $\mathrm{Tr}$}

Особенность логики $\operatorname{Tr}$ уже в том, что она единственная из всех рассмотренных четырехзначных логик, которая обладает интерполяционным свойством Крейга. Кроме того, она является прекрасным канди- 
датом на роль пропозициональной логики истины. Все эти вопросы мы рассмотрим в следующей статье, а здесь представим аксиоматизацию $\operatorname{Tr}$, где модальный оператор $\square$ есть эндоморфизм $g$ :

1. Классическая пропозициональная логика $\mathbf{C}_{2}$.

2. $\square(\varphi \rightarrow \psi) \leftrightarrow(\square \varphi \rightarrow \square \psi)$.

3. $\neg \square \varphi \leftrightarrow \square \neg \varphi$.

4. $\square \square \varphi \leftrightarrow \varphi$.

Правила вывода: modus ponens и правило Гёделя.

Доказательство полноты логики $\operatorname{Tr}$ по Крипке, а также доказательство алгебраической полноты будут представлены в следующей статье.

\section{Литература}

[1] Белнап H. Как нужно рассуждать компьютеру // Белнап H., Стил T. Логика вопросов и ответов. М.: Прогресс, 1981. С. 208-239.

[2] Белнап H. Об одной полезной четырехзначной логике // Белнап H., Cтил T. Логика вопросов и ответов. М.: Прогресс, 1981. С. 240-267.

[3] Бочвар Д.А., Финн В.К. О многозначных логиках, допускающих формализацию анализа антиномий. 1 // Исследования по математической лингвистике, математической логике и информационным языкам. М.: Наука, 1972. C. $238-295$.

[4] Вригт Г.Х., фон. Логика истины // Вригт Г.Х., фон. Логико-философские исследования. Избранные труды. М.: Прогресс, 1986. С. 555-579.

[5] Ермолаева Н.М., Мучник А.А. Модальные расширения логических исчислений типа Хао Вана // Исследования по формализованным языкам и неклассическим логикам. М.: Наука, 1974. С. 172-193.

[6] Ермолаева H.M., Мучник А.А. Модальные логики, определяемые эндоморфизмами дистрибутивных решеток // Исследования по теории множеств и неклассическим логикам. М.: Наука, 1976. С. 229-246.

[7] Ермолаева Н.М., Мучник А.А. Функционально замкнутые 4-значные расширения булевой алгебры и соответствующие логики // Исследования по неклассическим логикам и теории множеств. М.: Наука, 1979. С. 298-315.

[8] Карпенко А.С. Булевы каскады импликативных логик // Смирновские чтения: материалы 2-й международной научной конференции. М.: ИФРАН, 1999. C. $41-44$.

[9] Карпенко А.С. Развитие многозначной логики. М.: Издательство ЛКИ, 2010. $448 \mathrm{c}$. 
[10] Леммон Е. Алгебраическая семантика для модальных логик I / Семантика модальных и интенсиональных логик / Ред. В.А. Смирнов. М.: Прогресс, 1981. С. $98-124$.

[11] Леммон Е. Алгебраическая семантика для модальных логик II // Семантика модальных и интенсиональных логик / Ред. В.А. Смирнов. М.: Прогресс, 1981. С. $125-165$.

[12] Лукасевич Я. Аристотелевская силлогистика с точки зрения современной формальной логики. М.: Иностранная литература, 1959. 312 с.

[13] Попов B.M. Секвенциальные формулировки паранепротиворечивых логических систем // Синтаксические и семантические исследования неэкстенсиональных логик. М.: Наука, 1989. С. 285-289.

[14] Томова Н.Е. Импликативные расширения регулярных логик Клини // Логические исследования. 2010. Вып. 16. С. 233-258.

[15] Томова Н.Е. Естественные трехзначные логики: Функциональные свойства и отношения. М.: ИФРАН, 2012. 89 с.

[16] Эсакиа Л. Доказуемостные интерпретации интуиционистской логики // Логические исследования. 1998. Вып. 5. С. 19-24.

[17] Яблонский C.В. Функциональные построения в k-значной логике // Труды математического института им. В. А.Стеклова. 1958. Т. 51. С. 5-142.

[18] Coniglio M.E., Figallo M. Hilbert-style presentation of two logics associated to tetravalent modal algebras // Studia Logica. 2014. Vol. 102(3). P. 525-539.

[19] Font J.M. Belnap's four-valued logic and De Morgan lattices // Logic Journal of the IGPL. 1997. Vol. 5(3). P. 413-440.

[20] Font J.M., Rius M. An abstract algebraic logic approach to tetravalent modal logics // The Journal of Symbolic Logic. 2000. Vol. 65(2). P. 481-518.

[21] Karpenko A.S. Von Wright's truth-logic and around // Logical Investigations. 2013. Vol. 19. P. 39-50.

[22] Lewis C.I., Langford C.H. Symbolic Logic. N.Y.: Dover Publications, 1959 (2nd ed. with corrections). $506 \mathrm{p}$.

[23] Eukasiewicz J. A system of modal logic // The Journal of Computing Systems. 1953. Vol. 1. P. 111-149.

[24] Pynko A.P. Functional completeness and axiomatizability within Belnap's fourvalued logic and its expansion // Journal of Applied Non-Classical Logics. 1999. Vol. 9(1). P. 61-105.

[25] Sobochiński B. Modal system S4.4 // Notre Dame Journal of Formal Logic. 1964. Vol. 5(4). P. 305-312.

[26] Sobochiński B. Family K of the non-Lewis modal systems // Notre Dame Journal of Formal Logic. 1964. Vol. 5(4). P. 313-318.

[27] Zeman J.J. A study of some systems in the neighborhood of S4.4// Notre Dame Journal of Formal Logic. 1971. Vol. XII(3). P. 341-357. 


\title{
A.S. KARPENKO
}

\section{Lattices of Four-valued Modal Logics}

\author{
Karpenko Alexander Stepanovich \\ Department of Logic, Institute of Philosophy, Russian Academy of Sciences. \\ Volkhonka 14/5, Moscow, 119991, Russian Federation. \\ e-mail: as.karpenko@gmail.com
}

In this paper four lattices of four-valued modal logics are considered. Different algebraic structures are the basis for construction, then these structures are gradually extended by the endomorphisms and constant functions. In the first case, the lattice of extensions of Boolean algebra $B^{2}$ is formed, then the lattice of extensions of De Morgan algebra DM4 is constructed. In both cases the different modal logics appear, the properties of which are described and compared. The lattice with tetravalent modal logic TML is considered individually. Finally, the first two lattices are joined and the class of basic four-valued modal logics is singled out. This class consists of $\mathbf{E}$-modal system of Łukasiewicz, logic V2 of Sobochiński and von Wright's truth-logic $\mathbf{T}^{\prime \prime}$. Special attention should be paid to the logic $\mathbf{T r}$, which is functionally equivalent to the logic V2 and occupies a central place in the final lattice. This logic is the only of the above-considered logics which has the Craig's interpolation property, and besides is an excellent candidate for the role of propositional logic of truth. In conclusion its axiomatization is presented.

Keywords: modal logics, lattices of logics, Boolean algebra, De Morgan algebra, endomorphismus, closed classes of functions, Boolean cascades, logic $\mathbf{T r}$

\section{References}

[1] Belnap, N. "Kak nuzhno rassuzhdat' komp'yuteru" [How a computer should think], in: Belnap N. i Stil T., Logika voprosov $i$ otvetov [The logic of questions and answers]. Moscow: Progress, 1981, pp. 208-239. (In Russian)

[2] Belnap, N. "Ob odnoi poleznoi chetyrekhznachnoi logike" [On a useful fourvalued logic], in: Belnap N. i Stil T., Logika voprosov $i$ otvetov [The logic of questions and answers]. Moscow: Progress, 1981, pp. 240-267. (In Russian)

[3] Bochvar, D.A., Finn, V.K. "O mnogoznachnykh logikakh, dopuskayushchikh formalizatsiyu analiza antinomii. 1" [About many-valued logics, allowing the formalization of the analysis of antinomy. 1], in: Issledovaniya po matematicheskoi lingvistike, matematicheskoi logike i informatsionnym yazykam [Studies in mathematical linguistics, mathematical logic and language information]. Moscow: Nauka, 1972, pp. 238-295. (In Russian)

[4] Wright, G. H. Von. "Logika istiny" [The logic of truth], in: Wright, G. H. Von., Logiko-filosofskie issledovaniya. Izbrannye trudy [Logical and philosophical studies. Selected Works]. Moscow: Progress, 1986, pp. 555-579. (In Russian) 
[5] Ermolaeva, N. M., Muchnik, A. A. "Modal'nye rasshireniya logicheskikh ischislenii tipa Khao Vana" [Modal extensions of logical calculus such as Wang Hao], in: Issledovaniya po formalizovannym yazykam i neklassicheskim logikam [Studies on the formal language and non-classical logics]. Moscow: Science, 1974, pp. 172-193. (In Russian)

[6] Ermolaeva, N. M., Muchnik, A. A. "Modal'nye logiki, opredelyaemye endomorfizmami distributivnykh reshetok" [Modal logic determined by the endomorphismus of distributive lattices], in: Issledovaniya po teorii mnozhestv $i$ neklassicheskim logikam [Investigations on the theory of sets and non-classical logics]. Moscow: Science, 1976, pp. 229-246. (In Russian)

[7] Ermolaeva, N. M., Muchnik, A. A. "Funktsional'no zamknutye 4-znachnye rasshirenie bulevoi algebry i sootvetstvuyushchie logiki" [Functionally closed 4-valued extensions of Boolean algebra and the corresponding logics], in: Issledovaniya po neklassicheskim logikam i teorii mnozhestv [Investigations on non-classical logic and set theory]. Moscow: Science, 1979, pp. 298-315. (In Russian)

[8] Karpenko, A. S. "Bulevy kaskady implikativnykh logik" [Boolean cascades implicative logics], Smirnovskie chteniya [Smirnov's readings]. Proceedings of the Second International Scientific Conference. Moscow: IFRAS, 1999, pp. 4144. (In Russian)

[9] Karpenko, A. S. Razvitie mnogoznachnoi logiki [The development of multivalued logic]. Moscow: LCI Publisher, 2010. 448 pp. (In Russian)

[10] Lemmon, E. "Algebraicheskaya semantika dlya modal'nykh logik I" [Algebraic semantics for modal logics I], Semantika modal'nykh $i$ intensional'nykh logik [The semantics of modal and intensional logics], ed. V. A. Smirnov. Moscow: Progress, 1981, pp. 98-124. (In Russian)

[11] Lemmon, E. "Algebraicheskaya semantika dlya modal'nykh logik II" [Algebraic semantics for modal logics II], Semantika modal'nykh $i$ intensional'nykh logik [The semantics of modal and intensional logics], ed. V. A. Smirnov. Moscow: Progress, 1981, pp. 125-165.

[12] Lukasevich, Ya. Aristotelevskaya sillogistika s tochki zreniya sovremennoi formal'noi logiki [Aristotelian syllogistic from the poin of view of modern formal logic]. Moscow: Inostrannaya literatura, 1959, 312 pp. (In Russian)

[13] Popov, V.M. "Sekventsial'nye formulirovki paraneprotivorechivykh logicheskikh sistem" [Sequential formulations of paraconsistent logical systems], in: Sintaksicheskie $i$ semanticheskie issledovaniya neekstensional'nykh logik [Syntactic and semantic research of non-extensional logics]. Moscow: Nauka, 1989, pp. 285-289. (In Russian)

[14] Tomova, N.E. "Implikativnye rasshireniya regulyarnykh logik Klini" [Implicative extensions of regular Kleene logics], Logicheskie issledovaniya [Logical investigations], 2010, vol. 16, pp. 233-258. (In Russian) 
[15] Tomova, N.E. Estestvennye trekhznachnye logiki: Funktsional'nye svoistva $i$ otnosheniya [Natural three-valued logics: functional properties and relations], Moscow: IFRAN Publ, 2012, 89 pp. (In Russian)

[16] Esakia, L. "Dokazuemostnye interpretatsii intuitsionistskoi logiki" [Provableness interpretations of intuitionistic logic],Logicheskie issledovaniya [Logical investigations], 1998, vol. 5, pp. 19-24. (In Russian)

[17] Yablonskii, S.V. "Funktsional'nye postroeniya v k-znachnoi logike" [Functional constructions in k-valued logic], Trudy matematicheskogo instituta im. $V$. A.Steklova, 1958, vol. 51, pp. 5-142. (In Russian)

[18] Coniglio, M.E., Figallo, M. "Hilbert-style presentation of two logics associated to tetravalent modal algebras", Studia Logica, 2014, vol. 102(3), pp. 525-539.

[19] Font, J.M. "Belnap's four-valued logic and De Morgan lattices", Logic Journal of the IGPL, 1997, vol. 5(3), pp. 413-440.

[20] Font, J.M., Rius, M. "An abstract algebraic logic approach to tetravalent modal logics", The Journal of Symbolic Logic, 2000, vol. 65(2), pp. 481-518.

[21] Karpenko, A.S. "Von Wright's truth-logic and around", Logical Investigations, 2013, vol. 19, pp. 39-50.

[22] Lewis, C.I., Langford, C.H. Symbolic Logic. New York: Dover Publications, 1959 (2nd ed. with corrections). 506 pp.

[23] Łukasiewicz J. "A system of modal logic", The Journal of Computing Systems, 1953, vol. 1, pp. 111-149.

[24] Pynko, A.P. "Functional completeness and axiomatizability within Belnap's four-valued logic and its expansion", Journal of Applied Non-Classical Logics, 1999, vol. 9(1), pp. 61-105.

[25] Sobochiński, B. "Modal system S4.4", Notre Dame Journal of Formal Logic, 1964, vol. 5(4), pp. 305-312.

[26] Sobochiński, B. "Family K of the non-Lewis modal systems", Notre Dame Journal of Formal Logic, 1964, vol. 5(4), pp. 313-318.

[27] Zeman, J.J. "A study of some systems in the neighborhood of S4.4", Notre Dame Journal of Formal Logic, 1971, vol. XII(3), pp. 341-357. 